\title{
The etiology of diffuse cystic lung diseases: an analysis of 1010 consecutive cases in a LAM clinic
}

\author{
Han Cui ${ }^{1,2 \dagger}{ }^{+}$, Chongsheng Cheng ${ }^{1 \dagger}$, Wenshuai Xu' ${ }^{1}$, Xinlun Tian ${ }^{1}$, Yanli Yang ${ }^{1}$, Yani Wang ${ }^{1}$, Jiannan Huang ${ }^{1}$, \\ Yudi $\mathrm{He}^{1}$, Jun Wang ${ }^{1}$, Ruie Feng ${ }^{3}$, Weihong Zhang ${ }^{4}$ and Kai-Feng $\mathrm{Xu}^{1^{*}}$ (D)
}

\begin{abstract}
Background: The differential diagnosis of diffuse cystic lung disease (DCLD) is a clinical challenge. We wish to analyze the distribution of the etiology of DCLD based on data from a single lymphangioleiomyomatosis (LAM) clinic.

Methods: All DCLD patients at the LAM Clinic of Peking Union Medical College Hospital between January 2006 and December 2019 were analyzed. Information on the demographic, clinical, radiological, and pathological features was collected.

Results: A total of 1010 patients with DCLD on CT scan were evaluated. A sum of 711 (70.4\%) patients were diagnosed with definite or probable LAM. Other diagnoses included Birt-Hogg-Dubé syndrome (46), Sjogren's syndrome (38), pulmonary Langerhans cell histiocytosis (14), lung tumors (3), Castleman disease (2), antineutrophil cytoplasmic antibody-associated vasculitis (2), systemic lupus erythematosus (1), Marfan syndrome (1), amyloidosis (1), congenital cystic adenomatoid malformation of the lung (1), and pleuroparenchymal fibroelastosis (1). In the 38 patients diagnosed with Sjogren's syndrome, 2 were diagnosed with light-chain deposition disease, 2 were diagnosed with amyloidosis and 1 was diagnosed with lymphocytic interstitial pneumonia. One hundred and eighty-nine patients (18.7\%) were undiagnosed. Lung biopsy results were available in 27 patients in the undiagnosed DCLD group but did not provide a diagnosis.
\end{abstract}

Conclusion: Approximately 70\% of DCLD patients in our LAM clinic had LAM. The common differential diagnoses included Birt-Hogg-Dubé syndrome, Sjogren's syndrome, and pulmonary Langerhans cell histiocytosis. Detailed clinical information and laboratory, genetic, and pathological investigations provide correct diagnoses in most patients with DCLD.

Keywords: Diffuse cystic lung diseases, Etiology, Differential diagnosis, Lymphangioleiomyomatosis, Pulmonary Langerhans cell histiocytosis, Birt-Hogg-Dubé syndrome, Sjogren's syndrome

*Correspondence: xukf@pumch.cn

${ }^{\dagger} \mathrm{Han}$ Cui and Chongsheng Cheng have contributed equally to this work ${ }^{1}$ Department of Pulmonary and Critical Care Medicine, State Key

Laboratory of Complex Severe and Rare Diseases, Peking Union Medical College Hospital, Chinese Academy of Medical Sciences, Beijing, China

Full list of author information is available at the end of the article

\section{Introduction}

Diffuse cystic lung disease (DCLD) refers the presence of multiple air-filled spaces with a wall thickness less than $2 \mathrm{~mm}$ and clear boundaries with the lung tissues [1]. An increasing number of diseases labeled DCLD make the differential diagnosis of this condition difficult [2]. The clinical features and radiological findings are quite similar in many patients. An exploration of the 
appropriate diagnosis is a great clinical challenge. The aim of this study was to analyze the clinical data of DCLD patients registered in the Lymphangioleiomyomatosis (LAM) clinic of Peking Union Medical College Hospital (PUMCH) in recent years. We summarized the distribution of their etiologies and proposed a stepwise diagnostic procedure to improve the diagnosis of DCLD.

\section{Materials and methods Subjects}

All DCLD patients presented in the LAM Clinic of PUMCH between January 2006 and December 2019 were retrospectively analyzed. Their lung CT scans met the criteria for diffuse pulmonary cystic lesions: at least 3 air-filled spaces with a cyst wall less than $2 \mathrm{~mm}$, clearly demarcated from normal lung tissue. Patients with incomplete data were excluded. The study was approved by the Ethical Committee of PUMCH (S-K1583).

\section{Diagnosis}

The diagnostic criteria of LAM were initially based on the guidelines of the European Respiratory Society (ERS) in 2010 [3] and updated according to the 2017 guidelines by the American Thoracic Society and Japanese Respiratory Society (ATS/JRS) [4]. All definite LAM cases were reviewed and diagnosed based on the 2017 guidelines. Probable LAM was diagnosed based on the criteria of the 2010 guidelines, without other supporting evidence. The diagnoses of Sjogren's syndrome (SS) [5], Birt-HoggDubé syndrome (BHD) [6], pulmonary Langerhans cell histiocytosis (PLCH) [7], systemic lupus erythematosus [8], Castleman's disease [9], antineutrophil cytoplasmic antibody-associated vasculitis [10], and Marfan syndrome [11] were based on published criteria. Amyloidosis, light-chain deposition disease (LCDD), congenital cystic adenomatoid malformation of the lung, pleuroparenchymal fibroelastosis, and lung tumor were diagnosed based on pathology.

\section{Results}

\section{Patient population}

A total of 1076 patients with diffuse cystic changes on HRCT of the chest at the PUMCH LAM Clinic between January 2006 and December 2019 were included. Sixtysix subjects were excluded because of insufficient clinical information. A total of 1010 patients were finally analyzed in this article.

\section{Etiology}

In our LAM clinic, most subjects $(711 / 1010,70.4 \%)$ were diagnosed with LAM. Among them, the number of individuals with definite and probable LAM was 646 and 65, respectively. Most of the cases were sporadic LAM, and 58 were tuberous sclerosis (TSC)-associated LAM. Pathological results were obtained in $38.1 \%$ of LAM patients. The remaining 65 cases were probable LAM, 47 of them have serum vascular endothelial growth factor-D (VEGFD) tested and within normal ranges.

The number of SS patients was 38. Two patients were diagnosed with light-chain deposition disease (LCDD) and 2 patients were diagnosed with amyloidosis by videoassisted thoracic surgery (VATS) or transbronchial lung biopsy (TBLB). One patient was diagnosed with lymphocytic interstitial pneumonia (LIP) by VATS. There were 46 patients diagnosed with BHD, all of whom had folliculin $(F L C N)$ gene mutations. Fourteen patients were diagnosed with PLCH. These PLCH patients underwent VATS lung biopsy or TBLB, and the sample showed Langerhans cells. Three patients had lung tumors, and their pathology results were lung adenocarcinoma (1 patient), lymphangioma (1 patient), and unclassified spindle cell tumors (1 patient). Two patients had Castleman disease based on lung biopsy, clinical manifestations, and laboratory tests. Two patients were diagnosed with antineutrophil cytoplasmic antibody-associated vasculitis based on manifestations, lung CT findings, and positive for anti-neutrophil cytoplasmic antibody (ANCA). One patient had Marfan syndrome based on typical clinical features and a family history of Marfan syndrome and a mutation of $F B N 1$. One patient was diagnosed with systemic lupus erythematosus based on multisystemic involvement and positivity of anti-double-stranded DNA. One patient with amyloidosis, one patient with congenital cystic adenomatoid malformation of the lung, and one patient with pleuroparenchymal fibroelastosis were diagnosed by lung biopsy. For the other 189 patients, a definite diagnosis could not be made after several examinations. The distribution of etiologies is shown in Table 1. The top four causes of DCLD were LAM, BHD, SS, and PLCH, accounting for $80.1 \%$ of the causes of DCLD. There were $18.7 \%$ of patients undiagnosed.

\section{Basic information of the DCLD patients}

Clinical features including age, sex, smoking history, and family history among the different etiologies are shown in Table 2. Most patients were female at our LAM Clinic except for patients with PLCH. A total of 92.9\% (13/14) of PLCH patients had a smoking history. Family history was suggested in $1.1 \%(8 / 711)$ of LAM patients (all of them were TSC-associated) and in $80.4 \%$ (37/46) of BHD patients. 
Table 1 Etiology of diffuse cystic lung diseases

\begin{tabular}{ll}
\hline Causes & Numbers (\%) \\
\hline Lymphangioleiomyomatosis & $711(70.4 \%)$ \\
Birt-Hogg-Dubé syndrome & $46(4.6 \%)$ \\
Sjogren syndrome & $38(3.8 \%)$ \\
Light-chain deposition disease & 2 \\
Amyloidosis & 2 \\
Lymphocytic interstitial pneumonitis & 1 \\
Pulmonary Langerhans cell histiocytosis & $14(1.4 \%)$ \\
Tumor & $3(0.3 \%)$ \\
Castleman's disease & $2(0.2 \%)$ \\
Antineutrophil cytoplasmic antibody-associated vascu- & $2(0.2 \%)$ \\
litis & \\
Systemic lupus erythematosus & $1(0.1 \%)$ \\
Marfan syndrome & $1(0.1 \%)$ \\
Amyloidosis* & $1(0.1 \%)$ \\
Congenital cystic adenomatoid malformation of the lung & $1(0.1 \%)$ \\
Pleuroparenchymal fibroelastosis & $1(0.1 \%)$ \\
Undiagnosed diffuse cystic lung diseases & $189(18.7 \%)$ \\
Total & $1010(100 \%)$ \\
\hline
\end{tabular}

"This case of amyloidosis did not have Sjogren's syndrome

\section{Laboratory tests and other evaluations}

The characteristic clinical manifestations and laboratory tests are shown in Table 3. Among the patients diagnosed with LAM, 35.0\% had pneumothorax, 30.2\% had renal angiomyolipoma, $17.4 \%$ had chylothorax, and $3.9 \%$ had chylous ascites. Among patients with SS, 78.9\% experienced dry mouth, and $65.8 \%$ experienced dry eye. The positive rate of anti-SSA or anti-SSB antibody was $97.4 \%$. A total of $52.2 \%$ of BHD patients showed pneumothorax, and $17.4 \%$ showed skin involvement. The FLCN gene mutation were found in all BHD patients. Three patients with PLCH had diabetes insipidus, and magnetic resonance imaging of the head indicated pituitary funnel lesions. The patient with systemic lupus erythematosus showed symptoms with multisystem involvement and anti-dsDNA antibody positivity. The patient with Marfan syndrome was from a Marfan syndrome family, with a thin body habitus, long limbs, and an FBN1 gene mutation. One patient's laryngoscope revealed a larynx mass, and the pathology was amyloidosis. The patient with ANCA-associated vasculitis was ANCA positive. No

Table 2 Demographic characteristics among different causes of DCLD

\begin{tabular}{llllll}
\hline & Numbers & Age (years) & Female (\%) & Smoking history & Family history \\
\hline LAM & 711 & $38.2 \pm 10.8$ & $711(100.0 \%)$ & $1.1 \%$ & $1.1 \%$ \\
BHD & 46 & $47.3 \pm 10.4$ & $42(91.3 \%)$ & $6.5 \%$ & $80.4 \%$ \\
SS & 38 & $49.7 \pm 10.4$ & $38(100.0 \%)$ & $2.6 \%$ & 0 \\
PLCH & 14 & $32.8 \pm 12.4$ & $6(42.9 \%)$ & $92.9 \%$ & 0 \\
Tumor & 3 & $41-50$ & $3(100.0 \%)$ & 0 & 0 \\
CD & 2 & $34-46$ & $1(50 \%)$ & $50 \%$ & 0 \\
AVA & 2 & $37-51$ & $2(100 \%)$ & 0 & 0 \\
SLE & 1 & 45 & $1(100 \%)$ & 0 & 0 \\
MFS & 1 & 57 & 0 & 0 & 0 \\
AMY & 1 & 32 & $1(100.0 \%)$ & 0 & 0 \\
CCAM & 1 & 28 & $1(100.0 \%)$ & 0 & 0 \\
PPFE & 1 & 37 & $100.0 \%)$ & 0 & 0 \\
\hline
\end{tabular}

AMY amyloidosis, AVA antineutrophil cytoplasmic antibody-associated vasculitis, BHD Birt-Hogg-Dubé syndrome, CCAM congenital cystic adenomatoid malformation of the lung, CD Castleman disease, DCLD diffuse cystic lung disease, LAM lymphangioleiomyomatosis, MFS Marfan syndrome, PPFE pleuroparenchymal fibroelastosis, PLCH pulmonary Langerhans cell histiocytosis, SS Sjogren's syndrome

Table 3 Characteristic clinical manifestations and laboratory tests of DCLD

\begin{tabular}{lll}
\hline & Clinical manifestations & Evaluations \\
\hline LAM & $\begin{array}{l}\text { Pneumothorax 35.0\%, renal angiomyolipoma 30.2\%, chylothorax 17.4\%, chylous } \\
\text { ascites 3.9\%, TSC 8.2\% }\end{array}$ & VEGF-D $\geq 800$ pg/ml 89.0\% \\
SS & Dry mouth 78.9\%, dry eyes 65.8\% & Anti-SSA or anti-SSB antibody positive 97.4\% \\
BHD & Pneumothorax 52.2\%, fibrofolliculomas or trichodiscomas 17.4\% & FLCN gene mutation 100\% \\
PLCH & Pneumothorax 28.6\%, Diabetes insipidus 7.1\% & MRI showed pituitary funnel lesions 21.4\% \\
\hline
\end{tabular}

BHD Birt-Hogg-Dubé syndrome, LAM lymphangioleiomyomatosis, MRI magnetic resonance imaging, PLCH pulmonary Langerhans cell histiocytosis, SS Sjogren's syndrome, VEGF-D vascular endothelial growth factor-D 
clinical manifestation was recorded for the patients with congenital cystic adenomatoid malformation of the lung and pleuroparenchymal fibroelastosis.

\section{Undiagnosed DCLD}

One hundred eighty-nine patients (174 females, 15 males) were classified as having undiagnosed DCLD. Twelve of them had a smoking history. They underwent several examinations but still did not receive a diagnosis. Ten patients were consulted in the stomatology department without meaningful results. Ophthalmology tests were performed in 27 patients, and 5 patients were diagnosed with dry eye disease. Among the undiagnosed patients, serum protein electrophoresis (102 patients tested), immunofixation electrophoresis (123 tested), anti-SSA/SSB (135 tested), and ANCA (128 tested) were performed with negative results. Abdominal ultrasound was performed in 60 patients. Abdominal CT examination was performed in 45 patients, and kidney nodules were found in 1 patient. FLCN gene tests were performed in 29 patients, all of which were negative. One hundred forty-two VEGF-D examinations were performed, and all were lower than $800 \mathrm{pg} / \mathrm{ml}$. Twenty-six lung pathological biopsies were taken including 16 TBLB and 10 VATS. None of pathological results were suggestive. Among these 26 patients who underwent lung biopsy, 2 patients underwent $F L C N$ gene test with negative results.

\section{Discussion}

In this group, the most common causes of DCLD were LAM, SS, BHD, and PLCH, accounting for $80.1 \%$ of all causes. $18.7 \%$ were undiagnosed.

Research on the etiological distribution of DCLD is very limited; however, it is believed that the major causes of DCLD include LAM, PLCH, LIP, and BHD [12]. SS has not been listed sometimes because multiple pathological bases were found, such as LIP. Other causes of pathological abnormality in cystic lung lesion in patients with SS includes nonspecific interstitial pneumonia, usual interstitial pneumonia, organizing pneumonia, pulmonary lymphoma, pulmonary amyloidosis, and so on [13, 14]. Among the patients in this study, based on data available, 2 were diagnosed with amyloidosis, 2 were diagnosed with LCDD, and 1 was diagnosed with LIP. It seems that LIP was not the most common type of pathological abnormality.

Sex, smoking history, and family history are important in suggesting a differential diagnosis. Almost all LAM patients were women of childbearing age, and family history was mainly related to BHD and TSC. BHD is an autosomal-dominant genetic disease. A total of $80.4 \%$ of patients had a family history of pneumothorax or bullae in this report. A total of $57.1 \%$ of PLCH patients were male, and $92.9 \%$ of them had a history of smoking and no family history of similar diseases. All the SS patients were female in this report.

In the differential diagnosis of DCLD, characteristic changes in cysts in the lung can suggest a diagnosis clue of LAM type, BHD type, PLCH type, or others [2]. Laboratory investigations have mainly focused on autoimmune diseases such as SS and LCDD or amyloidosis. Recently, VEGF-D has been used in the diagnosis of LAM $[15,16]$. Gene tests, for single gene (FLCN, or TSC1/ TSC2), DCLD gene panel, or whole exome sequencing were selected depending on the aims of the test applied.

In our LAM clinic, LAM was the most common cause of DCLD. LAM can be diagnosed based on history (chylothorax, renal AML, or TSC), VEGF-D, and pathology [4]

Three patients in this group were diagnosed with tumors by a pathological diagnosis of their pulmonary cysts. Tumors that cause cystic lesions in the lung are mainly metastatic sarcoma and tumors derived from mesenchymal cells, including lung adenocarcinoma, bronchioloalveolar carcinoma, lymphoma, malignant vascular endothelial cell tumor, and osteosarcoma [17]. In this study, the pathology results of the 3 tumor patients were lung adenocarcinoma, lymphangioma and spindle cell tumor.

In this group, 189 patients had been labeled DCLD with unknown causes, accounting for a large proportion. Although they were screened initially, many of them lacked some tests, mainly either genetic screens or lung biopsies.

Based on the most common causes of DCLD, we proposed a stepwise diagnosis approach (Fig. 1). A detailed medical history, physical examination (sex, symptoms/ signs, smoking history, and family history) and pulmonary HRCT should be taken and analyzed to look for clues to common DCLD. Typical images of cysts on HRCT are suggestive of a diagnosis (Fig. 2), and are clues of further investigations. Further laboratory tests, gene tests and lung biopsy are frequently required. For undiagnosed DCLD, further diagnosis and follow-up are essential.

There are some limitations of our study. The patients in the LAM clinic were mainly female and showed signs suggestive of LAM; therefore, there was a selection bias among the patients in this report. The data represented were from only one clinic and, therefore, may not be representative of other populations. additionally, in the 


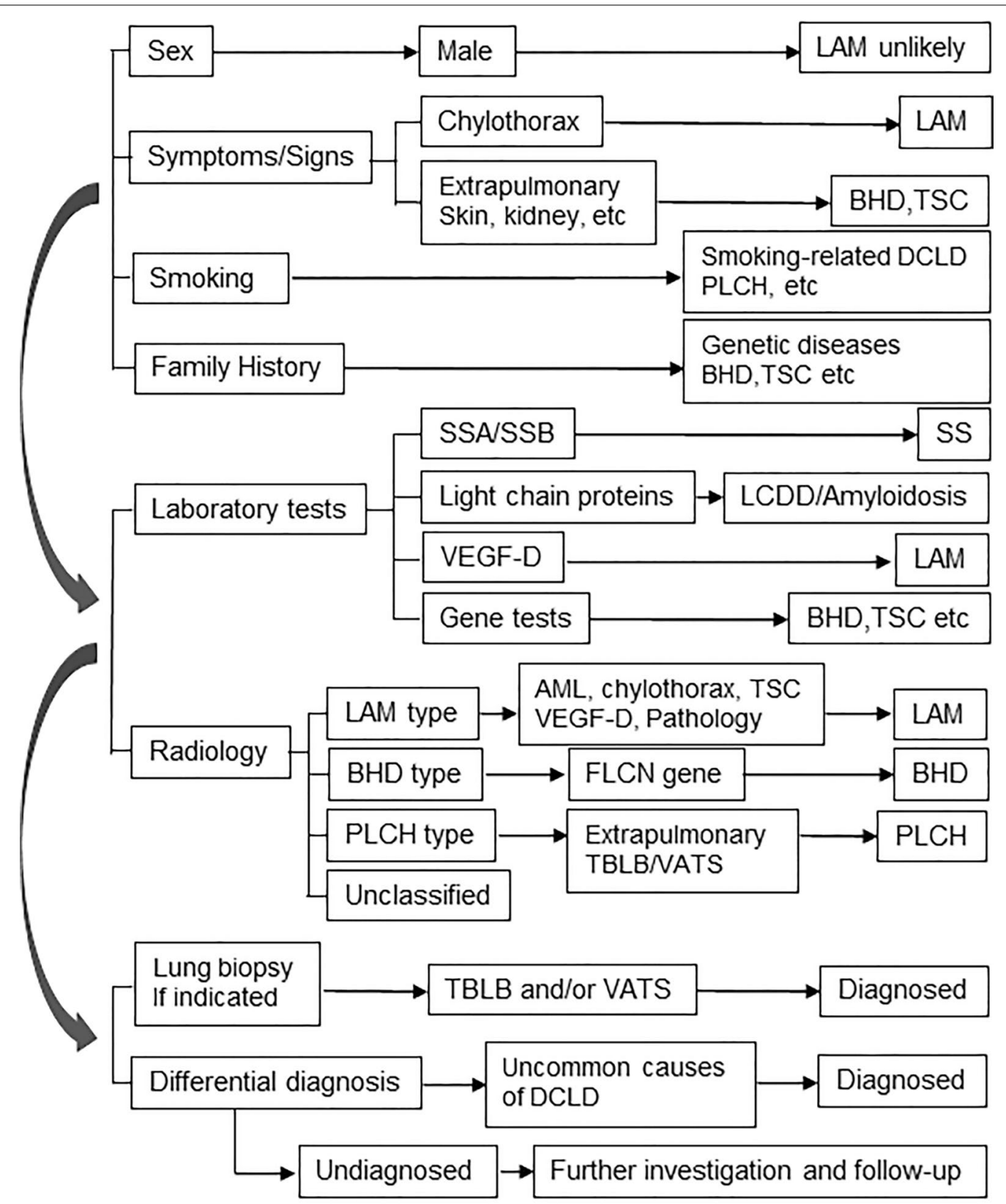

Fig. 1 Stepwise diagnostic procedure for common causes of diffuse cystic lung diseases. BHD Birt-Hogg-Dubé syndrome, FLCN folliculin, LAM lymphangioleiomyomatosis, LCDD light chain deposition disease, PLCH pulmonary Langerhans cell histiocytosis, SS Sjogren's syndrome, SSA/SSB Sjogren's syndrome antibodies A and B, TBLB transbronchial biopsy, TSC tuberous sclerosis complex, VATS video-assisted thoracoscopy, VEGF-D vascular endothelial growth factor-D

patients with undiagnosed DCLD, the gene tests and lung biopsy were inadequate.

We concluded that approximately $70 \%$ of DCLD patients presenting to the LAM clinic were diagnosed with LAM. Common causes in the differential diagnosis included SS, BHD, and PLCH. Detailed clinical information and laboratory, genetic, and pathological investigations can provide correct diagnoses in most patients with DCLD. 


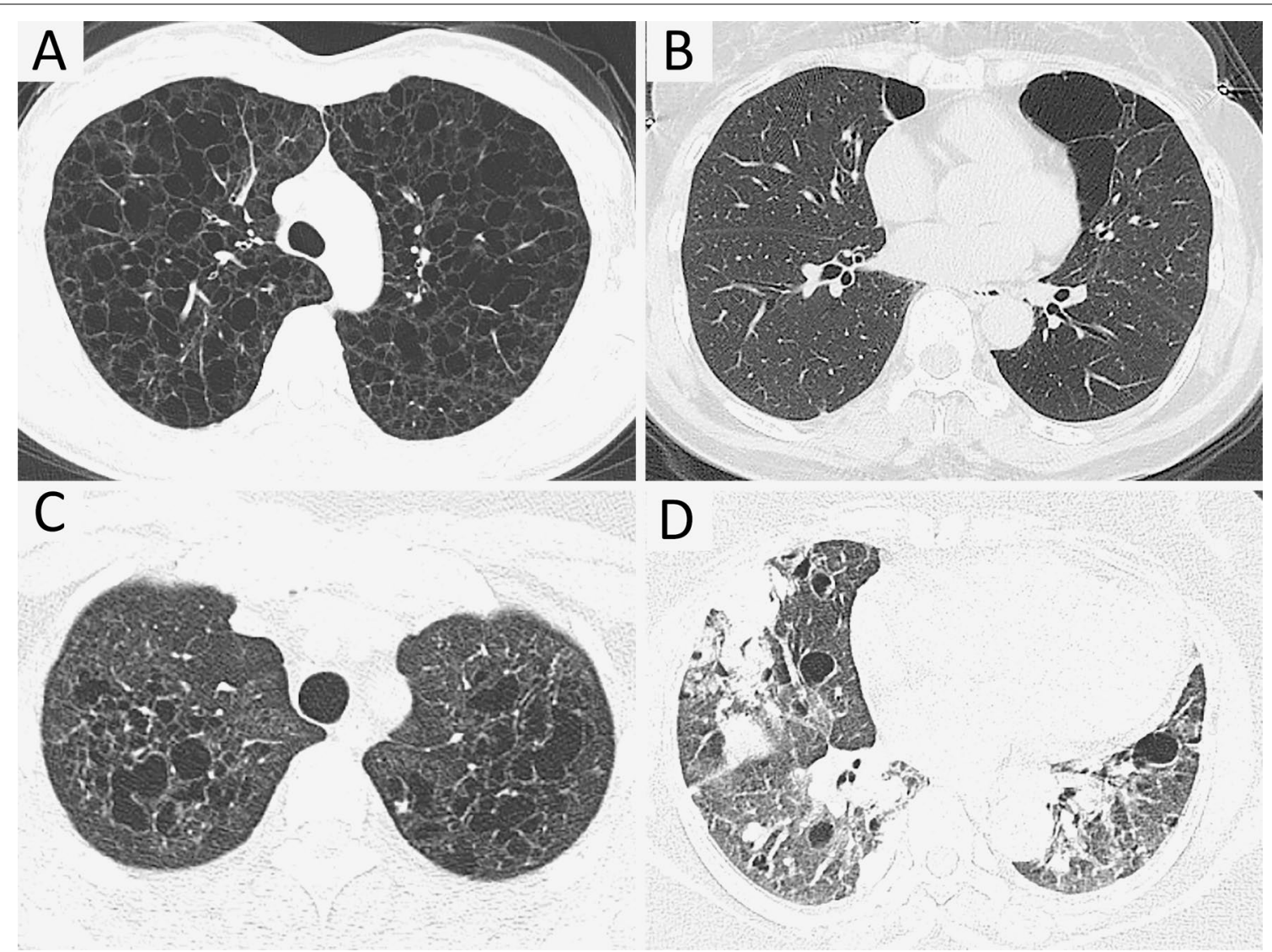

Fig. 2 Typical images of DCLD on CT scans are suggestive of a diagnosis. A A typical lymphangioleiomyomatosis (LAM) image in a 38-year-old woman shows a evenly distributed diffuse thin-walled cysts crossover all lung fields. Pneumothorax and chylothorax are frequently seen (not shown) in patients with LAM. (2) A typical Birt-Hogg-Dubé syndrome image in a 63-year-old woman shows large and irregular cysts frequently distributed in the lower lung fields and subpleural or paramediastinal regions. (3) A typical pulmonary Langerhans cell histiocytosis (PLCH) image in a 32-year-old woman with a history of cigarette smoking shows upper and middle lung field dominant distribution with irregular cysts and multiple nodules. Costophrenic regions are likely less involved (not shown). (4) A 66-year-old women with a history of Sjogren syndrome was found diffuse cystic changes in the lung. Images on CT show randomly distributed cysts that are larger than cysts usually presented in LAM as well as patchy infiltrates and nodules. Light chain deposition disease was diagnosed after thoracoscopic lung biopsy

\section{Abbreviations}

AML: angiomyolipoma; AMY: amyloidosis; ANCA: anti-neutrophil cytoplasmic antibody; AVA: antineutrophil cytoplasmic antibody-associated vasculitis; BHD: Birt-Hogg-Dubé syndrome; CCAM: congenital cystic adenomatoid malformation of the lung; CD: Castleman disease; DCLD: diffuse cystic lung disease; LAM: lymphangioleiomyomatosis; LCDD: light chain deposition disease; LIP: lymphocytic interstitial pneumonia; MFS: Marfan syndrome; MRI: magnetic resonance imaging; PPFE: pleuroparenchymal fibroelastosis; PLCH: pulmonary Langerhans cell histiocytosis; PUMCH: Peking Union Medical College Hospital; SS: Sjogren's syndrome; SSA/SSB: Sjogren's syndrome antibodies A and B; TBLB: transbronchial lung biopsy; TSC: tuberous sclerosis complex; VATS: videoassisted thoracic surgery; VEGF-D: vascular endothelial growth factor-D.

\section{Acknowledgements}

The authors thank all patients who participated in this study.

\section{Authors' contributions}

HC, CC: data collection, analysis, and manuscript writing. WX: data collection and analysis. XT, YY, YW, JH, YH, JW: data collection. RF: data collection (pathology). WZ: data collection (radiology). KFX: design, data collection, analysis, and manuscript writing. All authors read and approved of the final version of the manuscript.

\section{Funding}

This work was supported by the National Natural Science Foundation of China (U20A20341), the CAMS Innovation Fund for Medical Sciences (CIFMS) (2017-I2M-2-001), and the National Key Research and Development Program of China (2016YFC0901502).

\section{Availability of data and materials}

The dataset used in this research and analysis were available from the corresponding authors.

\section{Declarations}

\section{Ethics approval and consent to participate}

The protocol of this retrospective analysis was approved by the Ethical Committee of Peking Union Medical College Hospital (S-K1583). All patients provided written informed consent.

\section{Consent for publications}

Consent for publications were obtained from all participants. 


\section{Competing interests}

The authors declare no potential conflicts of interest with respect to the research, authorship, and publication of this article.

\section{Author details}

${ }^{1}$ Department of Pulmonary and Critical Care Medicine, State Key Laboratory of Complex Severe and Rare Diseases, Peking Union Medical College Hospital, Chinese Academy of Medical Sciences, Beijing, China. ${ }^{2}$ Department of Pathophysiology, State Key Laboratory of Medical Molecular Biology, Institute of Basic Medical Sciences, Chinese Academy of Medical Sciences, Peking Union Medical College, Beijing, China. ${ }^{3}$ Department of Pathology, Peking Union Medical College Hospital, Chinese Academy of Medical Sciences, Beijing, China. ${ }^{4}$ Department of Radiology. Peking, Union Medical College Hospital, Chinese Academy of Medical Sciences, Beijing, China.

Received: 6 January 2021 Accepted: 7 June 2021

Published online: 12 June 2021

\section{References}

1. Hansell DM, Bankier AA, MacMahon H, McLoud TC, Muller NL, Remy J. Fleischner Society: glossary of terms for thoracic imaging. Radiology. 2008:246(3):697-722.

2. Xu KF, Feng R, Cui H, Tian X, Wang H, Zhao J, et al. Diffuse cystic lung diseases: diagnostic considerations. Semin Respir Crit Care Med. 2016;37(3):457-67.

3. Johnson SR, Cordier JF, Lazor R, Cottin V, Costabel U, Harari S, et al. European respiratory society guidelines for the diagnosis and management of lymphangioleiomyomatosis. Eur Respir J. 2010;35(1):14-26.

4. Gupta N, Finlay GA, Kotloff RM, Strange C, Wilson KC, Young LR, et al. Lymphangioleiomyomatosis diagnosis and management: high-resolution chest computed tomography, transbronchial lung biopsy, and pleural disease management. An official American Thoracic Society/Japanese Respiratory Society clinical practice guideline. Am J Respir Crit Care Med. 2017;196(10):1337-48

5. Vitali C, Bombardieri S, Jonsson R, Moutsopoulos HM, Alexander EL, Carsons SE, et al. Classification criteria for Sjogren's syndrome: a revised version of the European criteria proposed by the American-European Consensus Group. Ann Rheum Dis. 2002;61(6):554-8.

6. Menko FH, van Steensel MA, Giraud S, Friis-Hansen L, Richard S, Ungari S, et al. Birt-Hogg-Dube syndrome: diagnosis and management. Lancet Oncol. 2009;10(12):1199-206.
7. Girschikofsky M, Arico M, Castillo D, Chu A, Doberauer C, Fichter J, et al. Management of adult patients with Langerhans cell histiocytosis: recommendations from an expert panel on behalf of Euro-Histio-Net. Orphanet J Rare Dis. 2013;8:72.

8. Petri M, Orbai AM, Alarcon GS, Gordon C, Merrill JT, Fortin PR, et al. Derivation and validation of the systemic lupus international collaborating clinics classification criteria for systemic lupus erythematosus. Arthritis Rheum. 2012;64(8):2677-86.

9. Fajgenbaum DC, Uldrick TS, Bagg A, Frank D, Wu D, Srkalovic G, et al. International, evidence-based consensus diagnostic criteria for HHV-8-negative/idiopathic multicentric Castleman disease. Blood. 2017;129(12):1646-57.

10. Jennette JC, Falk RJ, Bacon PA, Basu N, Cid MC, Ferrario F, et al. 2012 revised international Chapel Hill consensus conference nomenclature of vasculitides. Arthritis Rheum. 2013;65(1):1-11.

11. Loeys BL, Dietz HC, Braverman AC, Callewaert BL, De Backer J, Devereux $\mathrm{RB}$, et al. The revised Ghent nosology for the Marfan syndrome. J Med Genet. 2010:47(7):476-85.

12. Ryu JH, Tian X, Baqir M, Xu K. Diffuse cystic lung diseases. Front Med. 2013;7(3):316-27.

13. Stojan G, Baer AN, Danoff SK. Pulmonary manifestations of Sjogren's syndrome. Curr Allergy Asthma Rep. 2013;13(4):354-60.

14. Shi JH, Liu HR, Xu WB, Feng RE, Zhang ZH, Tian XL, et al. Pulmonary manifestations of Sjogren's syndrome. Respiration. 2009;78(4):377-86.

15. Xu KF, Zhang P, Tian X, Ma A, Li X, Zhou J, et al. The role of vascular endothelial growth factor- $D$ in diagnosis of lymphangioleiomyomatosis (LAM). Respir Med. 2013;107(2):263-8.

16. Young LR, Vandyke R, Gulleman PM, Inoue Y, Brown KK, Schmidt $\mathrm{LS}$, et al. Serum vascular endothelial growth factor-D prospectively distinguishes lymphangioleiomyomatosis from other diseases. Chest. 2010;138(3):674-81.

17. Gupta N, Vassallo R, Wikenheiser-Brokamp KA, McCormack FX. Diffuse cystic lung disease. Part I. Am J Respir Crit Care Med. 2015;191(12):1354-66.

\section{Publisher's Note}

Springer Nature remains neutral with regard to jurisdictional claims in published maps and institutional affiliations.
Ready to submit your research? Choose BMC and benefit from:

- fast, convenient online submission

- thorough peer review by experienced researchers in your field

- rapid publication on acceptance

- support for research data, including large and complex data types

- gold Open Access which fosters wider collaboration and increased citations

- maximum visibility for your research: over 100M website views per year

At BMC, research is always in progress.

Learn more biomedcentral.com/submissions 